Loaiza-Aguirre, M. I., y Andrade-Abarca, P. S. (mayo-agosto, 2021). Análisis de programas de desarrollo profesional del profesorado universitario. Revista Virtual Universidad Católica del

Norte, (63), 161-195. https://www.doi.org/10.35575/rvucn.n63a7

\title{
Análisis de programas de desarrollo profesional del profesorado universitario
}

\author{
Analysis of professional development programs for university faculty
}

\author{
María Isabel Loaiza-Aguirre \\ Doctora en Innovación Educativa y Aprendizaje a lo largo de la vida \\ Vicerrectorado Académico, Universidad Técnica Particular de Loja \\ Loja, Ecuador \\ miloaiza@utpl.edu.ec
}

Orcid: http://orcid.org/0000-0002-5217-1325

\section{Paola Salomé Andrade-Abarca}

Máster en Innovación y Desarrollo de Competencias en la Educación Superior

Vicerrectorado Académico, Universidad Técnica Particular de Loja

Loja, Ecuador

psandrade@utpl.edu.ec

Orcid: http://orcid.org/0000-0003-1129-8597

Recibido: 01 de julio de 2020

Evaluado: 09 de octubre de 2020

Aprobado: 24 de febrero de 2021

Tipo de artículo: Investigación Científica y Tecnológica

\section{Resumen}

Los programas de desarrollo profesional se diseñan considerando elementos enfocados en cómo ayudar al profesorado universitario en los ámbitos: docencia, investigación y gestión; atendiendo las necesidades e intereses identificados, actividades, etapas de la formación, evaluación y transferencia. El objetivo de esta investigación fue analizar los programas de desarrollo profesional ofertados en un grupo de instituciones de educación superior -IES- de Ecuador y diagnosticar las acciones que se han implementado en este contexto. Para recopilar la información se utilizó la entrevista, organizando los datos a través de un sistema de registro categorial, con cuatro 
categorías: (1) Contexto; (2) Planificación y diseño; (3) Desarrollo y puesta en marcha; y, (4) Seguimiento y gestión de la calidad. Los resultados más relevantes mostraron que los programas de desarrollo profesional son fragmentados en cursos aislados, sin responder a objetivos comunes; pocas universidades mantienen una estructura integral. Se concluyó que la institucionalización de las acciones de formación es un elemento que debe considerarse en los planes estratégicos de las universidades, integrándolos a las necesidades, para gestionar políticas que motiven al profesorado a valorar la importancia de su desarrollo profesional. Los hallazgos contribuyen al ámbito del desarrollo profesional docente, aportando un análisis integral frente a estudios específicos.

Palabras clave: Desarrollo profesional; Educación superior; Formación de profesores; Profesor Universitario; Universidad.

\footnotetext{
Abstract

Professional development programs are designed considering elements focused on how to help university faculty in the areas: teaching, research and management; attending to the identified needs and interests, activities, stages of training, evaluation and transfer. The objective of this research was analyzed the Professional Development Programs offered in a group of Higher Education Institutions in Ecuador and diagnose the actions that have been implemented in this context. To collect the information, the interview was used, organizing the data through a Categorial Registry System with four categories: (1) Context; (2) Planning and Design; (3) Development and Start-up; and, (4) Monitoring and Quality Management. The most relevant results showed that Professional Development Programs are fragmented into isolated courses without responding to common objectives, few universities maintain an integral structure. The study concluded that the institutionalization of training actions is an element that must be considered in the strategic plans of the universities, integrating them to the needs to manage policies that motivate teachers to value the importance of their professional development. The findings contribute to the field of teacher professional development, providing a comprehensive analysis against specific studies.
} 
Keywords: Development professional; Higher education; Teacher training; University professor; University.

\section{Introducción}

Transformar el sistema educativo, permitiendo el acceso a la educación y ofertando una formación de calidad, es uno de los ideales más importantes presentes en la última década en los países latinoamericanos. Esta preocupación está presente en el Ecuador, en donde el acceso a la educación superior ha aumentado un 59 \%, en los más recientes años, pasando de 443,509 a 703,806 estudiantes matriculados (Restrepo y Stefos, 2017). Este incremento es uno de los resultados de varias iniciativas orientadas a lograr esta transformación, considerada como la base para el desarrollo de la matriz productiva del país y enfocada en lograr una disminución en el índice de pobreza y desigualdad social.

Para mejorar la calidad de la educación superior, el profesorado es un actor que interviene, tanto en el proceso de enseñanza como en el aprendizaje de los estudiantes, por lo que su desarrollo profesional y pedagógico tiene un especial interés. Esta preocupación ha cobrado atención en el contexto de la educación del Ecuador, sobre todo en la última década; es así que, en la Ley Orgánica de Educación Superior (LOES) 0/2010, de 12 de octubre, emitida por la Asamblea de la República del Ecuador; en el Reglamento de Carrera y Escalafón del Profesor e Investigador, del Sistema de Educación Superior, expedido el 31 de octubre de 2012 por el Consejo de Educación Superior (CES); en el Reglamento de Régimen Académico, de noviembre del 2013 aprobado por el CES; y en el Modelo de Evaluación Institucional, definido el 20 de noviembre del 2013 por el Consejo de Evaluación, Acreditación y Aseguramiento de la Calidad de la Educación Superior (CEAACES), se presenta, en varios apartados, la necesidad de que el sistema de educación superior ecuatoriano cuente con una planta de docentes capacitados y que participen continuamente en procesos de formación.

En el Reglamento de Carrera y Escalafón del Profesor e Investigador, del Sistema de Educación Superior vigente en este país, se establece que el profesorado universitario deberá contar con un nivel de formación, mínimo de maestría, o su equivalente para poder ser docente universitario. A partir de esta disposición se ha observado un incremento del $29 \%$, en el 2008, al 
$58 \%$ en el 2014, de profesores universitarios con formación de maestría o PhD (Restrepo y Stefos, 2017).

Además del requisito de título de cuarto nivel, en el mencionado Reglamento se determina respecto a la promoción del docente en las diferentes categorías y niveles, el cumplimiento de horas de capacitación y actualización profesional, tanto en metodologías de aprendizaje e investigación, como en relación al área de conocimiento del profesorado, según sus actividades de docencia o investigación.

Esto significa que las universidades ecuatorianas deben considerar promover la formación de sus docentes en ejercicio, tomando en cuenta que, para el 2015, apenas un $10 \%$ de los profesores que laboran en instituciones de educación superior tenía alguna formación en pedagogía, concluyéndose que un 90 \% del profesorado en ejercicio debería recibir algún tipo de preparación para la docencia (Fabara Garzón, 2016).

Los docentes juegan un papel importante como los principales agentes de cambio en la metodología, la didáctica y la pedagogía, para reelaborar las relaciones entre el estudiante y el conocimiento (Cardeño Espinosa et al., 2017, p. 65). La profesión de la enseñanza exige tanto a las universidades como al profesorado un trabajo conjunto y una participación permanente en los procesos de formación. "El aprendizaje continuo y permanente no es ya una opción a elegir, sino una obligación moral para una profesión comprometida con el conocimiento" (Marcelo, 2007, p. 2). Por tanto, es indispensable la formación docente en nuevas competencias profesionales e innovaciones tecnológicas, que deben combinarse con las habilidades transversales, como el razonamiento crítico, la capacidad de trabajar en equipo, la buena comunicación verbal y escrita, el desarrollo de la creatividad y las llamadas competencias socio-emocionales (Martínez Villalobos et al., 2016).

Frente a este reto, la presente investigación analiza la interrogante: ¿Cómo se están desarrollando los programas de desarrollo profesional del profesorado universitario? Hasta la fecha, en Ecuador, no se han realizado estudios en relación a esta temática, por lo que este trabajo puede ser referencia para futuras investigaciones en este ámbito. Además, puede contribuir con diferentes indicadores encaminados a orientar propuestas de las diferentes acciones de formación. 


\section{Marco teórico}

Hay muchos elementos en relación con el desarrollo profesional de profesores en el ámbito universitario; para sustentar la presente investigación se partirá de un análisis de su importancia, principales criterios y elementos fundamentales, considerando que en el caso de las instituciones de educación superior, estas deben emprender diferentes acciones enfocadas a constituirse en agentes de cambio; para esto, es necesario entender el rol del docente con un perfil transformador y desarrollar diferentes esfuerzos, enfocados al desarrollo profesional del profesorado.

\section{Sentido e importancia del desarrollo profesional del profesorado universitario}

Las organizaciones, no únicamente educativas, tienen una responsabilidad social con el entorno, por lo que deben participar continuamente en procesos de formación que les permitan ser más innovadoras y creativas; para lograr esto, deben prestar vital atención al capital humano y a su desarrollo profesional (Gómez, 2009).

La expresión desarrollo profesional corresponde a términos como formación permanente, formación continua, formación en servicio, desarrollo de recursos humanos, aprendizaje a lo largo de la vida, reciclaje o capacitación, entre otros; sin embargo, la noción de desarrollo profesional es el que se adapta mejor a la concepción del docente como profesional de la enseñanza (Vaillant, 2016).

Villa (2001) define al desarrollo profesional del profesorado como "un proceso de crecimiento, formación y mejora en el ejercicio profesional" (p. 173), por lo que destaca la formación como un elemento del desarrollo profesional. Además, Melo \& Campos (2019) presentan al desarrollo profesional como un proceso de autoentrenamiento y entrenamiento colectivo, es decir, conformado por la formación del propio docente y el aprendizaje desarrollado a través del equipo de profesores, que ocurre debido a necesidades reales, contextualizadas en el espacio de tiempo del trabajo de los docentes, de forma que el conocimiento y la práctica permiten al profesorado desarrollarse y mejorar su desempeño laboral. Por tanto, el desarrollo docente del profesorado se presenta como un conjunto de factores que posibilitan, impiden o favorecen que el profesorado progrese en el ejercicio de su trabajo (Imbernón, 2020). 
En la actualidad se reconoce, en las universidades del mundo, a la formación docente como una herramienta necesaria para el desarrollo profesional del profesorado y como un elemento fundamental para la mejora de la calidad docente (Feixas et al., 2013); todo esto refleja, sin duda, la relación entre el desarrollo profesional del docente universitario y su formación, por lo que es fundamental su estudio.

El desarrollo profesional del docente es resultado de un conjunto de fases, con la presencia de factores familiares, políticos, sociales, culturales, que pueden facilitar u obstaculizar el proceso de formación (Boéssio y Portella, 2009); sin embargo, el resultado de estas experiencias dependerá en alta medida del propio compromiso e interés del profesor.

Los programas de desarrollo profesional prestan atención a la motivación propia del profesorado; en la figura 1 se presenta el modelo propuesto por Lipowsky (2011), y adaptado por Richter et al. (2019), en el cual se muestra que la participación del profesorado en acciones de formación depende de variables relacionadas con el contexto, la institución y la propia motivación; por tanto, no se puede hablar de crecimiento ni de desarrollo, si no existe el interés y la motivación de las personas por la formación (Fernández March, 2009; Ruè, 2015). Sobre esta misma reflexión, Knight (2005) manifiesta que los motivos intrínsecos y las oportunidades de realización y actualización en el ámbito laboral, se constituyen en fuentes importantes de motivación para el profesorado.

\section{Figura 1}

Elementos considerados por el profesorado en acciones de formación

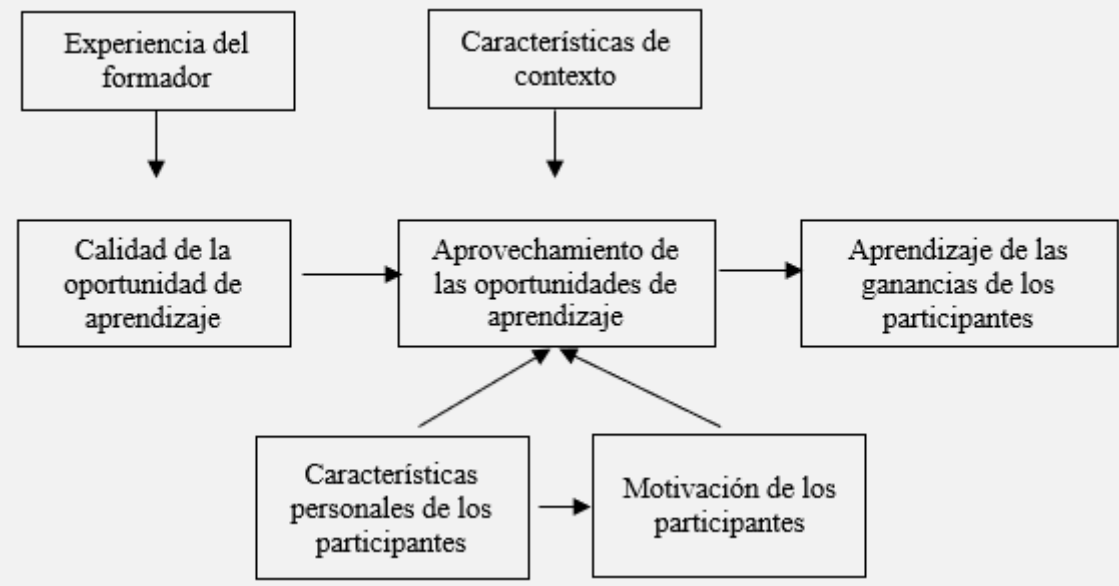

Nota: Adaptado de Richter et al. (2019). 
Algunos estudios han analizado los elementos que motivan la participación de los docentes en acciones de formación, y la relación entre estos elementos con las características personales. Richter et al. (2019) concluyen que los elementos más comunes que motivan al profesorado son el interés personal y mejorar la práctica docente; mientras que los elementos que generan una menor motivación son la promoción en la carrera y el reconocimiento social; el contacto social genera una motivación media. Adicional a esto, indican que la motivación de los maestros disminuye a medida que aumenta los años de experiencia.

Aparte de la motivación del profesorado por participar en actividades orientadas a su desarrollo profesional, es fundamental de analizar al profesor en su rol de persona. Korthagen (2010) resalta la importancia de tener en cuenta todas las dimensiones de la persona, considerando su misión e identidad como profesor y, a la vez, sus características profesionales y personales.

Además de los elementos mencionados, Cabrera (2002) indica que: el avance del conocimiento, los avances de la tecnología y la velocidad del cambio son factores que inciden en las necesidades del desarrollo profesional.

Paralelo a estos, se pueden distinguir dos factores adicionales que justifican la importancia y necesidad del desarrollo profesional; por un lado, está el factor económico y demográfico, ya que se requieren estructuras organizativas más flexibles y descentralizadas que permitan afrontar los cambios económicos acaecidos, lo que exige, a su vez, una polivalencia por parte de los empleados y/o miembros de la organización; así como que el envejecimiento progresivo de las plantillas hace que la formación sea indispensable para hacer frente a los constantes cambios contextuales. Otro factor para considerar son las innovaciones tecnológicas, ya que alteran significativamente el ámbito ocupacional, aumentando la demanda de personal más cualificado (Parellada et al., 1999, como se citó en Gómez, 2009, p. 114).

Para incorporar en las reflexiones sobre la formación de los profesores a los aspectos personales de la enseñanza, se propone el "modelo cebolla", que es una adaptación del "Modelo de Bateson" (Korthagen, 2004). En este, se integran seis elementos: el entorno, los estudiantes, competencias, creencias subyacentes, identidad del docente y, un sexto, reflexionando la misión que tiene la persona como profesor (ver figura 2). 


\section{Análisis de}

\section{Figura 2}

Modelo cebolla (Korthagen, 2004)

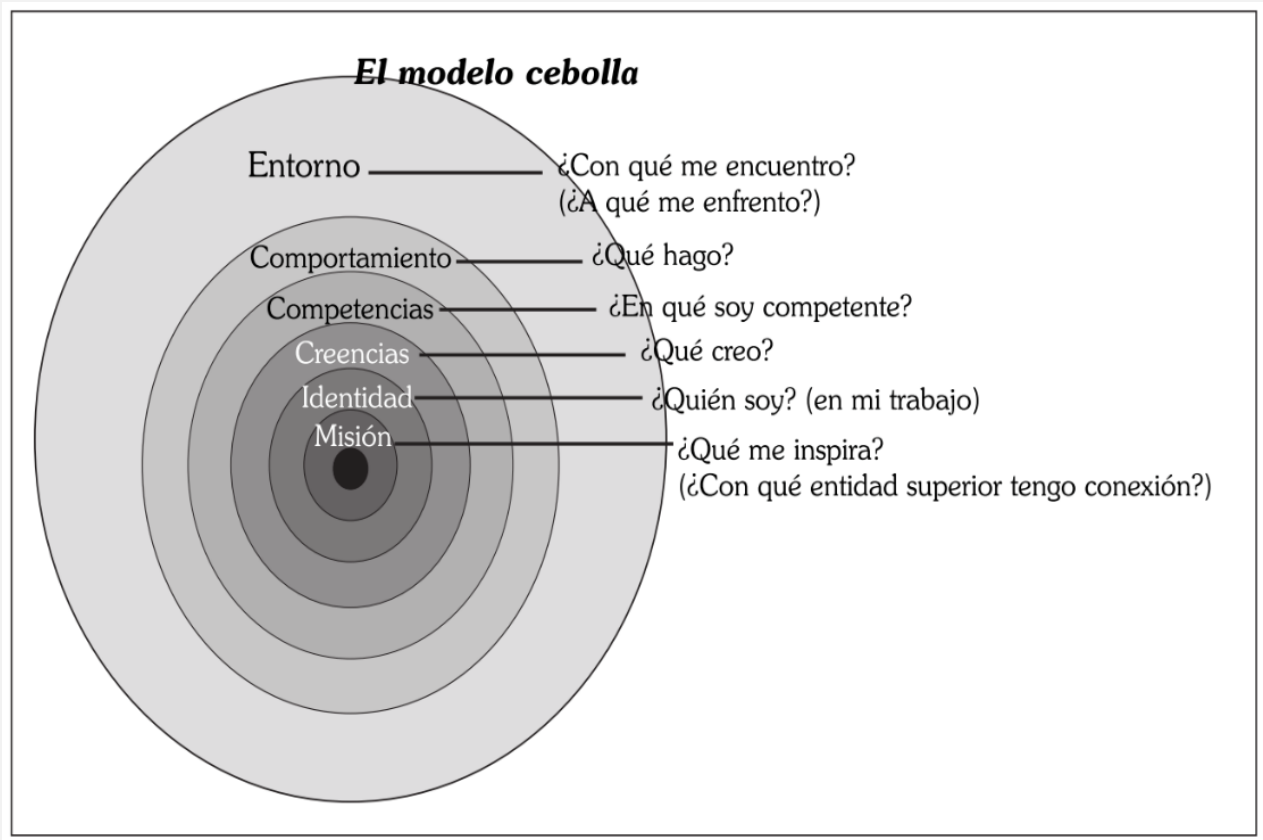

Nota: Adaptado de Korthagen (2004).

Esta propuesta se caracteriza por la relación entre todos los niveles. Los ideales del profesor corresponden a sus actuaciones y comportamientos en las situaciones específicas de la enseñanza, y están relacionadas con el entorno en donde se desarrollan; además, forma parte fundamental el compromiso del profesor frente a los estudiantes y a la institución, lo que permite proyectar el desarrollo profesional con la inclusión de técnicas o modelos que orienten el desarrollo de las cualidades esenciales para ser docente; por ello, todos estos aspectos deben ser considerados al momento de hablar de formación y desarrollo profesional del profesorado.

Además, hay que considerar la motivación y orientación del profesor por la carrera de la enseñanza, por lo que, como parte de su desarrollo profesional se deben incluir actividades que fomenten la autodeterminación y su motivación intrínseca, orientadas a conseguir objetivos claros en el proceso de enseñanza aprendizaje (Saavedra Bautista, 2018). 


\section{Criterios para el desarrollo profesional del profesorado universitario}

Las siguientes interrogantes, planteadas por Ruè (2015), pueden servir como un punto de partida para analizar el desarrollo profesional del profesorado: ¿de qué manera puede ser mejorado el proceso de enseñanza y aprendizaje?, ¿cómo los profesores pueden trabajar en dicha mejora? Las respuestas no son sencillas y dependerán, entre otros aspectos, de cómo se concibe el proceso de enseñanza aprendizaje en cada institución, las necesidades y estrategias de mejora para cubrir este proceso y los contextos culturales relacionados con el profesorado, y que, sin duda, deben ser concebidos como criterios para su desarrollo profesional. Este ha sido un ámbito de reflexión de varios autores, quienes han propuesto líneas de actuación para orientar a las instituciones de educación superior a enfocar la formación de su profesorado de manera efectiva (De la Torre, 2000; Imbernón, 2011; Tejada Fernández, 2000; Zabalza, 2013).

En la década de los 90, Guasti (como se citó en Zabalza, 2011b) propuso un esquema que resume los ejes del cambio o criterios que debían considerarse al estructurar los modelos de formación: i) Formación centrada en la clase (frente a la centralidad de la disciplina); ii) Refuerzo de los vínculos entre formación - investigación; iii) Diferenciación de las funciones (en la medida que van apareciendo nuevos roles en el ejercicio profesional); y iv) Red de servicios de apoyo.

Hoy en día, aunque se hable de transformación en las universidades, estos puntos siguen siendo muy relevantes; la actualización realizada a estos enfoques corresponde al primer punto, ya que actualmente la formación está centrada en el aprendizaje del estudiante y no en la clase (Zabalza, 2011b).

Al referirse a la enseñanza centrada en el estudiante, se establece una relación dinámica entre la calidad del proceso de enseñanza y la profesionalización docente. Estudios señalan que existen mejores resultados de calidad de enseñanza cuando el profesor está continuamente involucrado en actividades de formación (López, 2005). De esta manera, para el desarrollo profesional del profesorado se pueden considerar las dimensiones de: (a) perfeccionamiento permanente, (b) análisis de la práctica y (c) investigación en el aula.

El perfeccionamiento permanente del profesorado tiene una relación directa con su capacidad de aprender a aprender a lo largo de la vida y además se vincula con la implicación de 
la institución y la motivación personal para involucrarse y ser activo en su desarrollo profesional. El análisis de la práctica y la investigación en el aula coinciden también con la preocupación de varios autores por reforzar, a través de acciones de formación, el vínculo entre la docencia e investigación; en este sentido, López (2005) incluye, en su propuesta, la autorreflexión del propio docente, la reflexión del equipo de docentes y de los responsables de la formación.

Day \& Sachs (2004), resumen estos tres criterios básicos que se deben considerar durante el desarrollo profesional del profesorado: (a) alinear la práctica docente con las políticas educativas, (b) mejorar los resultados del alumnado a través de una mejora de la actividad docente, y (c) mejorar el estatus y el perfil de la profesión docente.

De la misma forma, la participación del profesorado en procesos de investigación de su práctica docente es una función cada vez más importante en el contexto actual, y que debe considerarse al momento de hablar de su formación, ya que además de facilitador y promotor de aprendizajes significativos, el docente debe participar en actividades de investigación, lo que le permitirá potenciar su desarrollo profesional y su práctica en el aula (Reis-Jorge et al., 2020).

Paralelo a esto, en los procesos de formación del profesorado debe fortalecerse la apropiación de los docentes sobre el manejo y utilidad de las TIC en el proceso de enseñanza, incluyendo el dominio de herramientas tecnológicas en la práctica diaria y acciones orientadas al fomento de la innovación (Lopera et al., 2021). Además, deberá existir una interconexión entre la pedagogía, didáctica y tecnología, favoreciendo la formación del docente, por medio de un aprendizaje mediado por la virtualidad (Roldán, 2018).

Otro criterio importante como marco de referencia para el desarrollo profesional del profesorado universitario, es que se debe considerar su perfil profesional, existiendo diferentes niveles y escenarios de actuación, por ejemplo: profesor titular, profesor asociado, profesor colaborador, tutor; con base en esto se deben definir perfiles requeridos para poder plantear la formación (Tejada Fernández, 2013).

Por otra parte, al momento de hablar de criterios para la formación del docente universitario, se debe considerar el aprendizaje previo que tiene el profesorado, ya que desarrollan patrones mentales y creencias sobre la enseñanza, a partir de la larga etapa de observación que experimentan como estudiantes (Marcelo y Vaillant, 2009) originándose el aprendizaje informal. Los futuros profesores construyen su aprendizaje con base en modelos y experiencias con los que 
se sienten mayormente identificados, sin cuestionarse la utilidad del método, influyendo más los aspectos emocionales que los racionales. Este conocimiento informal, es el primer tipo de conocimiento que tiene el profesorado, y sobre este partirá su desarrollo profesional, logrando adaptar, desarrollar, cambiar o desechar las creencias y sus conocimientos. Las experiencias del profesorado, como estudiantes, son importantes para evaluar el nuevo conocimiento y analizar qué es compatible con las creencias y conceptos desarrollados (Kane et al., 2002).

Varios autores analizados (Day, 2005; De Ketele, 2003; De la Torre, 2000; Fernández March, 2008; Mateo, 2012; Mayor et al., 2003; Mayor Ruiz, 2007) coinciden en algunos criterios básicos que pueden orientar la elaboración de una propuesta de formación, señalando los siguientes: a) metas y planes estratégicos de la institución, b) perfil de competencias del docente, c) modelo académico en el que se basa la formación de los estudiantes, d) estudio de las necesidades de formación del profesorado, e) resultados de evaluación del profesorado, f) diagnóstico de enfoques de enseñanza del profesorado y enfoques de aprendizaje de los estudiantes, g) diagnóstico de los métodos de enseñanza más comunes, y h) estrategias que fomenten la investigación e innovación pedagógica.

El enfoque de enseñanza del profesorado y los métodos utilizados deberían diagnosticarse al inicio de cualquier acción de formación. Se entiende como enfoques de enseñanza "a los planes y estrategias que los docentes implementan en su enseñanza y se interpretan como la manera en la que los docentes enseñan" (Postareff, Lindblom-Ylanne, 2007, como se citó en Yunga et al., 2016, p. 316). De forma general estos enfoques se dividen en: centrados en el profesor y en el estudiante; en el primero, se prioriza la transmisión unidireccional de la información docente - alumno, considerado como el método tradicional en el que el alumno es un simple receptor de la información; en el segundo, el docente motiva el proceso de aprendizaje del estudiante, favoreciendo la construcción de su propio conocimiento. Tomando en cuenta esto, se podrán plantear acciones de formación que ayuden al profesor a desarrollar el enfoque de su formación, establecido en la institución, inclinado hacia el aprendizaje de los estudiantes.

Mateo (2012) propone considerar los resultados de la evaluación del profesorado como otro de los criterios al momento de diseñar los programas de desarrollo profesional del profesorado, refiriéndose a la actividad individual y colectiva, tanto en docencia como en investigación. Estos resultados pueden servir para proponer acciones de formación orientadas a la 
mejora y a la innovación, enfocándolas a mejorar el proceso de enseñanza y los resultados de aprendizaje de los estudiantes (De Ketele, 2003).

Se debe tener presente dos posibles extremos que se pueden presentar al momento de plantear acciones de formación sobre los resultados de evaluación del profesorado, considerando que la invitación a participar de estas acciones estará dirigida, entre otros docentes, a quienes registraron resultados de evaluación por debajo del valor admitido como "bueno" en la institución, por lo que, dentro de este grupo, por un lado, estarán los profesores creativos, dinámicos, quienes aprovecharán la formación como una actividad de mejora y desarrollo profesional; y en el otro lado, están los profesores que verán estas actividades como auténticas “cárceles”, ya que no sienten la necesidad o presentan desconfianza de participar en los procesos de cambio. "La invitación a ser desarrollado profesionalmente puede considerarse como recordatorios de las insuficiencias presentes" (Day, 2005, p. 128).

\section{Elementos presentes al momento de hablar de desarrollo profesional del profesorado}

En forma general, los elementos deben considerar las necesidades y cambios que la institución se ha planteado como retos, y las necesidades de formación del profesorado, tomando en cuenta que la actividad docente es una profesión que requiere de formación para acreditar las competencias necesarias para su ejercicio (Fernández March, 2006). A partir de estos elementos se establecerán los objetivos, contenidos, estrategias metodológicas y el sistema de evaluación de las formaciones.

Al referirse a la institución, se considerarán las políticas institucionales, condiciones contextuales, organizativas y culturales en las que se llevan a cabo las acciones de formación; el compromiso con la calidad docente que tenga la universidad, el cual deberá estar contemplado en los planes estratégicos y planes de mejora; y las políticas de incentivación y acreditación de la calidad de la docencia.

En lo que tiene que ver con la profesionalización de la docencia, se deben tomar en cuenta elementos como la selección de contenidos formativos, resultados de aprendizaje esperados, y perfil de competencias del profesorado, haciendo referencia en las dimensiones pedagógicas, institucionales y socio profesionales (Fernández March, 2008). 
Además, se deberá tener presente la congruencia existente entre las acciones de formación del profesorado y la consecución del perfil de egreso de los estudiantes, el cual es establecido formalmente en las titulaciones. La mayoría de los programas de formación para el desarrollo profesional del profesorado considera elementos que están relacionados con los diferentes momentos en los que se desarrolla el proceso de enseñanza (Yániz Álvarez, 2008, p. 11): (a) planificación de la docencia con el enfoque de diseño curricular; (b) comprensión de los procesos de aprendizaje y el adecuado tratamiento de los elementos más influyentes; (c) metodologías de problemas, casos y proyectos; y (d) evaluación como componente formativo del currículo y las técnicas de evaluación auténtica.

La integración de los diferentes elementos responderá al diseño de una propuesta de desarrollo profesional del profesorado, que se oriente a la mejora de la calidad del proceso de enseñanza - aprendizaje que se imparte en las instituciones de educación superior, su transferencia a la sociedad y la propia satisfacción del profesorado.

\section{Consideraciones sobre los modelos de desarrollo profesional del profesorado universitario}

Estudios sobre el desarrollo profesional del profesorado reconocen cuatro etapas claves a la hora de plantear estrategias que impacten en el aula: fases previas de los aspirantes a docentes, formación inicial en una institución específica, primeros años de ejercicio profesional y a la formación continua (Vaillant, 2016).

El modelo de formación propuesto por Tejada Fernández y Navío Gámez (2007), presentan varios aspectos que coinciden con la lógica de la contextualización del currículo y el desarrollo del proceso de enseñanza, al igual que lo plantea Yániz Álvarez (2008). Se puede resumir que las acciones de formación se desarrollan mediante ciclos; previo a la planificación de la formación se deben tener presentes las necesidades, las cuales están representadas por diferentes actores y dependen del contexto y de las metas institucionales. Posteriormente, se inicia con la planificación en la que se determinan objetivos, contenidos, estrategias a desarrollar para lograr la comprensión y el aprendizaje del profesorado, y se proponen las herramientas para el seguimiento y evaluación permanente. Finalmente, se valoran los resultados, se elabora el plan de mejoras y se inicia nuevamente el ciclo de formación, teniendo presente que el modelo es flexible y debe responder a 
los cambios y exigencias que se presentan durante el camino de la formación. En resumen, lo que estos autores señalan es que las etapas de los programas de desarrollo profesional del profesorado deben considerar: (a) diagnóstico de las necesidades de formación y desarrollo profesional detectadas, (b) planificación de las acciones de formación y herramientas de seguimiento y evaluación, y (c) evaluación del programa y planteamiento de mejoras.

Algunas de las características consideradas en el diseño del programa de formación son: duración, temática, horas presenciales y virtuales, y tipos de actividades de aprendizaje; sin embargo, Kennedy (2016) manifiesta que estas características pueden ser predictores poco confiables del éxito del programa; el autor enfatiza la importancia del trabajo con comunidades de aprendizaje, la calidad de entrenadores, intensidad del programa, pero sobre todo sugiere reemplazar la concepción actual de los programas de formación, pasando del conjunto de características presentes en el diseño a una concepción que integra el quehacer del profesorado, sus motivaciones e intereses, cómo aprenden y cómo se desarrollan profesionalmente.

En lo que tiene que ver a las modalidades de formación, se han identificado que son muy diversas en los modelos de formación, y se las puede clasificar en: estudios universitarios (postgrados, por ejemplo); cursos (suelen responder a necesidades puntuales); trabajo cooperativo entre el profesorado (reciben distintas denominaciones: formación entre iguales, recíproca, etc.); seminarios y foros (preferentemente sobre temas de actualidad); jornadas y encuentros con amplia participación (actualización en temas pedagógicos); talleres de reflexión y círculos de estudio en los centros; asesoramiento pedagógico en los departamentos y centros; lo anterior, desde las modalidades de formación, relacionadas con la presencialidad, la semipresencialidad y la virtualidad (Imbernón et al., 2011). Además, para estos mismos autores estos programas de formación consideran, en lo referente al modo de participación: individual o colectiva; nivel de planificación de la actividad: bajo un proyecto o no, planificación cerrada o no; nivel de interacciones entre participantes: organizadores, expertos, asesores, participantes; grado de implicación y autonomía; dinámica y estructura interna de las sesiones, entre otros.

Respecto a las instancias ejecutoras de los programas de desarrollo profesional docente, mientras en algunos modelos son preferentemente las facultades las que integran en sus estructuras organizativas un departamento de educación encargado de la formación docente, en otros, la formación se lleva a cabo a través de unidades centralizadas que actúan con respecto a todas las 
facultades y escuelas, con base en las demandas específicas de algunas facultades concretas (Zabalza, 2011a)

Es común que en los modelos de desarrollo profesional se incorporen acciones de seguimiento y evaluación como una fase de las fases de formación, con el fin de determinar diferentes problemas durante su desarrollo. Además, la medición de la transferencia de los resultados de las formaciones y el impacto logrado en la institución será un indicador de calidad de las acciones de formación.

En lo que tiene que ver a la evaluación de los planes de formación, se han identificado cuatro grandes dimensiones: el plan de formación, el proceso de implementación de dicho plan, el nivel de satisfacción de los implicados, y el impacto de la formación (Zabalza, 2011a). Para este mismo autor, cada una de estas dimensiones son valoradas por un criterio de evaluación que orientará el juicio de valor y se concretan en preguntas o cuestiones.

El impacto de la formación docente suele ser relacionado con su transferencia, entendida como la aplicación efectiva y continua de un conjunto de habilidades, conocimientos y concepciones, aprendidas en un contexto de desarrollo docente (Feixas et al., 2013).

\section{Metodología}

\section{Estudio de caso universidades ecuatorianas}

El Sistema de Educación Superior del Ecuador actualmente está conformado por universidades y escuelas politécnicas, de tipo públicas, privadas y cofinanciadas; institutos tecnológicos superiores públicos e institutos privados, de acuerdo con lo descrito en la figura 3. 


\section{Figura 3}

Tamaño del Sistema de Educación Superior del Ecuador

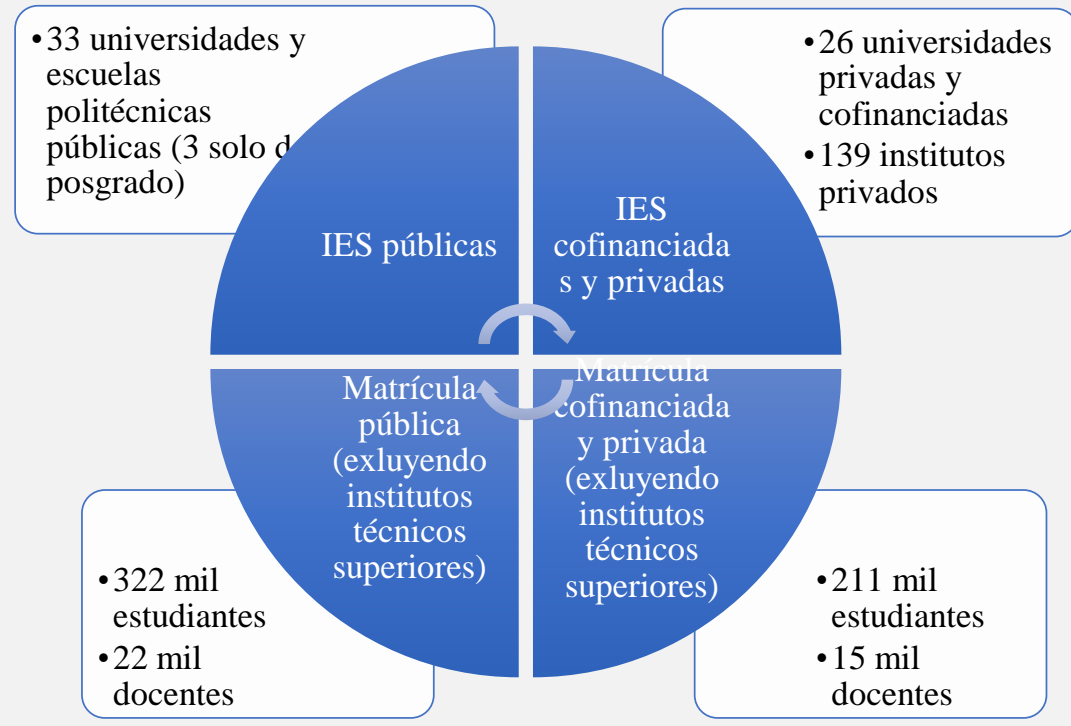

Nota: Tomado de Herdoíza (2015).

Según la Secretaría Nacional de Educación Superior Ciencia, Tecnología e Innovación SENESCYT- (2018), existen 51 instituciones de educación superior en el Ecuador; estas ofertan un total de 629 carreras universitarias. De acuerdo con su categoría, las universidades se ubican en cuatro escalas descritas en la tabla 1:

\section{Tabla 1}

Clasificación de las universidades que ofertan grado y posgrado de Ecuador según la categoría

\begin{tabular}{cc}
\hline Categoría & Nro. de instituciones que ofertan grado y posgrado ${ }^{1}$ \\
\hline Categoría A & 3 \\
Categoría B & 22 \\
Categoría C & 18 \\
Categoría D & 8 \\
\hline
\end{tabular}

Nota: Tomado de SENESCYT (2018).

\footnotetext{
${ }^{1}$ En esta clasificación no se incluyen la Universidad de Investigación Experimental Yachay-Tech, Universidad de las Artes, Universidad Nacional de Educación y la Universidad Regional Amazónica, ya que son instituciones públicas creadas recientemente.
} 


\section{Población y muestra}

La población elegida para la presente investigación corresponde a las universidades de categoría A y B de Ecuador, dado que cumplen, como mínimo, con el $45 \%$ de los indicadores de calidad exigido por el CEAACES.

En un primer acercamiento se envió la solicitud de colaboración para participar en este estudio a todas las universidades ubicadas en estas categorías. Se recibió la confirmación de la participación de nueve de estas, lo que significa el $36 \%$ de la población. Algunas universidades que no participaron de la investigación presentaron varias justificaciones, entre estas se mencionan: necesidad de cumplir con algunos procesos burocráticos para contar con la autorización respectiva que permita al personal de la universidad ofrecer la información, por lo que el tiempo de respuesta impediría continuar con la planificación de la investigación; poca disponibilidad de tiempo del personal; y, en algunas instituciones de educación superior, por disposiciones internas no está permitido ofrecer la información solicitada. También, existió un porcentaje inferior que no emitió ningún tipo de respuesta a la invitación.

Las nueve universidades que participaron en la investigación tienen concordancia con lo expuesto por Leandro da Silva y Tejada Fernández (2016), sobre criterios de conocimiento previo de la institución de educación superior, establecimiento de contacto con algunos informantes clave, respuesta positiva de los gestores universitarios y por la facilidad de acceso a los datos. Estas universidades se ubican geográficamente en las ciudades con mayor número de habitantes de Ecuador: Guayaquil, Quito y Cuenca.

\section{Tipo de estudio, variables y procedimiento}

Se emplearon estudios descriptivos con el propósito de presentar algunas características de los elementos que integran la oferta de los programas de desarrollo profesional en las universidades analizadas.

El estudio se organizó en tres fases: (1) revisión documental de la información disponible en las webs de las universidades, referente a los programas de desarrollo profesional; (2) 
entrevistas al personal responsable; (3) revisión documental de ciertas políticas, manuales y procedimientos de cada universidad; y (4) análisis y discusión de resultados.

Para el estudio se utilizó el modelo procesual integrado por cuatro dimensiones, propuesto por Marcelo (2007), quien desarrolló estándares para someter a evaluación de calidad las diferentes fases que integran un programa de desarrollo profesional. Aunque este modelo tiene un enfoque para la modalidad de aprendizaje abierto y a distancia, fue diseñado para "ser lo suficientemente flexible como para acomodarse a los diferentes formatos que puede adoptar la formación docente" (Marcelo y Zapata, 2008, p. 25). De las seis dimensiones propuestas en el modelo, se consideraron las de mayor relación con entornos presenciales: (1) Contexto; (2) Planificación y diseño; (3) Desarrollo y puesta en marcha; y, (4) Seguimiento y gestión de la calidad.

De acuerdo con la metodología seleccionada, se aplicó el procedimiento descrito en la tabla 2.

\section{Tabla 2}

Modelo procesual para evaluación de la calidad de un Programa de Desarrollo Profesional

\begin{tabular}{lll}
\hline \multicolumn{1}{c}{ Dimensión } & \multicolumn{1}{c}{ Procedimiento } \\
\hline (1) Contexto & $\begin{array}{l}\text { Revisión de los planes estratégicos y planes operativos } \\
\text { anuales de las nueve instituciones de educación superior } \\
\text { participantes. } \\
\text { Análisis de contextos, en lo referente a las existencias de } \\
\text { normativa y políticas de formación. }\end{array}$ \\
\hline (2) Planificación y diseño & $-\begin{array}{l}\text { Análisis de las necesidades, objetivos, contenidos, } \\
\text { actividades, recursos y medios que se consideran para la } \\
\text { ejecución de un programa de desarrollo profesional. }\end{array}$ \\
\hline $\begin{array}{ll}\text { (3) Desarrollo y puesta en } \\
\text { marcha }\end{array}$ & $\begin{array}{l}\text { Análisis de los procesos de difusión y orientaciones dadas } \\
\text { al profesorado sobre la formación. }\end{array}$ \\
\hline (4) Seguimiento y gestión de & $-\begin{array}{l}\text { Mecanismos de seguimiento y evaluación de los programas } \\
\text { de desarrollo profesional. }\end{array}$ \\
la calidad & $\begin{array}{l}\text { Creación de espacios comunes para el intercambio de } \\
\text { experiencias y recursos útiles para el proceso de enseñanza. }\end{array}$ \\
\hline
\end{tabular}
Nota: elaboración propia, a partir de Marcelo (2007).

Durante la fase de recolección de datos, varios informantes, haciendo referencia a las acciones de formación, mencionaron términos como curso, programa, plan; que "desde una perspectiva óptica representan distintos niveles operacionales” (Tejada Fernández y Navío Gámez, 


\section{Análisis de}

2007, p. 159). Un curso ofrece formación en una temática específica y concreta; un programa se diseña con el objeto de cumplir necesidades formativas, y puede incluir uno o varios cursos; y, un plan hace referencia a las directrices, políticas de la institución, y puede ser integrado y desarrollado por diferentes programas (Institute of Personal and Development, 2000; Tejada Fernández y Navío Gámez, 2007). A pesar de que el enfoque de cada una de estas perspectivas es diferente, en el análisis de los resultados no se realizarán distinciones, ya que la denominación suele solaparse; además, los elementos investigados son comunes en todas las perspectivas de formación (Institute of Personal and Development, 2000).

\section{Recogida de los datos}

Para recoger los datos se utilizaron diferentes informantes, según la técnica de investigación utilizada (ver tabla 3).

\section{Tabla 3}

Técnicas utilizadas e informantes

\begin{tabular}{cc}
\hline Técnica & Informante \\
\hline Análisis documental & $\begin{array}{c}\text { Fuentes documentales: documentos oficiales de las } \\
\text { instituciones de educación superior internos y externos }\end{array}$ \\
\hline Entrevista & $\begin{array}{c}\text { Se realizaron nueve entrevistas a directores, coordinadores o } \\
\text { responsables de los programas de desarrollo profesional del } \\
\text { profesorado en las instituciones de educación superior. }\end{array}$ \\
\hline
\end{tabular}

Nota: Elaboración propia.

\section{Fuentes documentales}

Para realizar el análisis de la información, se revisaron documentos oficiales de las universidades: 


\section{Material interno}

Se revisó la normativa de todas las nueve universidades que participaron en el estudio, analizando políticas, planes, procedimientos, manuales y/o informes que aluden directa 0 indirectamente a la formación del profesorado. A estos documentos se accedió en forma digital por medio de la información publicada en internet o en forma física, por medio de los documentos recolectados para el análisis. Los documentos analizados fueron: (a) 9 planes estratégicos, (b) 9 planes de mejora y rendición de cuentas, (c) 9 planes operativos anuales, (d) 7 políticas, reglamentos o instructivos sobre capacitación, perfeccionamiento o desarrollo personal docente, y (e) 7 programas o planes de formación.

\section{Material externo}

Este tipo de material corresponde a los documentos elaborados por la institución, que son de acceso público y sirven para comunicarse con el exterior: revistas, comunicaciones, cartas y divulgaciones (Del Rincón y otros, 1995, como se citó en Tapia Henríquez, 2013).

Se realizó la búsqueda de la información sobre las distintas actividades relacionadas con los programas de desarrollo profesional en las páginas web de las universidades. Además, en el caso de estar disponible, se revisó la información publicada de las unidades o centros de formación propios de cada universidad.

\section{Dimensiones consideradas para el análisis documental y entrevistas}

Para organizar la información se diseñó un sistema de registro con cuatro dimensiones: (1) Contexto; (2) Planificación y diseño; (3) Desarrollo y puesta en marcha; y (4) Seguimiento y gestión de la calidad. Estas permiten analizar en forma procesual las fases que atraviesa un programa de desarrollo profesional (Marcelo y Zapata, 2008). Dependiendo de la amplitud de la dimensión, algunas se dividieron en subdimensiones. Cada una de estas, agrupa varios estándares, los cuales fueron diseñados tomando como referencia el modelo propuesto por Marcelo y Zapata 


\section{Análisis de}

(2008). De los estándares propuestos en este modelo, se adaptaron los que guardan relación con los elementos analizados.

\section{Resultados y discusión de los datos}

En la dimensión (1) Contexto, se analizó la existencia de normativa y políticas que evidencien la implicación de cada institución en las acciones de formación. Se analizó la existencia de estas acciones con las diferentes denominaciones dadas en cada universidad, y se enumeró las dependencias que tienen la responsabilidad de gestionarlas.

Se observó que el fortalecimiento pedagógico del profesorado está presente dentro de los planes estratégicos y planes operativos anuales (POA) de las nueve instituciones analizadas. Estas declaraciones indican que las universidades tienen presente la necesidad y la importancia de generar políticas que incentiven, promuevan y apoyen la participación del profesorado en acciones de formación pedagógica, lo que da cumplimiento a lo establecido en el Reglamento de Régimen Académico, de noviembre del 2013 aprobado por el CES. Esto es congruente con lo mencionado por Zabalza (2011a), quien menciona que la formación docente es un elemento institucional, que se constituye en una acción de la universidad en su conjunto, y compromete, por tanto, a los equipos rectorales y a los planes estratégicos de mejora institucional que propongan.

En esta misma dimensión, al analizar las diferentes acciones que se consideran dentro de la formación, se identificó que todas las instituciones de educación superior diseñan y gestionan programas de desarrollo profesional o planes de formación integrados por cursos, talleres o capacitaciones (ver figura 4). 


\section{Figura 4}

Acciones de formación que integran los programas de desarrollo profesional

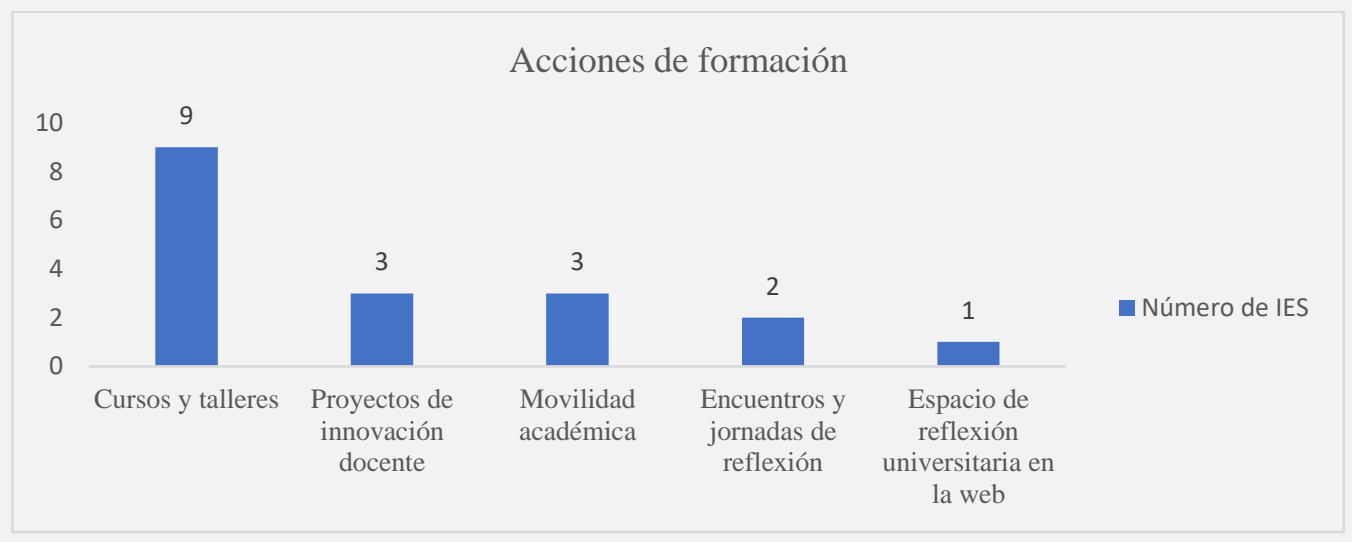

Nota: Elaboración propia.

Todas las instituciones de educación superior analizadas diseñan y gestionan planes o programas de formación, integrados por cursos, talleres o capacitaciones. La denominación dada a estas acciones es muy variada: Programas de perfeccionamiento de la docencia universitaria y de capacitación docente, Ciclo básico de formación docente, Plan de actualización y capacitación docente, Formación y capacitación del cuerpo docente, Actualización y perfeccionamiento docente, Programa de formación docente, Programas de perfeccionamiento de la docencia universitaria y de capacitación docente, Sistema de Capacitación/autoformación docente y personal de apoyo académico, Programa de formación docente.

En todas las instituciones de educación superior existen sistemas con mayor o menor estructura que se encargan de la formación pedagógica del profesorado; esto depende del tamaño de la institución y/o de los recursos disponibles para disponer de un departamento específico de formación, entre otros factores; de cualquier manera, el interés principal se enfoca en ofrecer el apoyo necesario y respuesta a las necesidades concretas de formación o cualificación para el desarrollo profesional del profesorado (Tejada Fernández y Navío Gámez, 2007), y no concretamente a dar cumplimiento a la normativa existente o los objetivos puntuales de las dependencias que gestionan la formación.

De manera general, se identificó que las cuatro universidades que gestionan las acciones de formación por medio de direcciones/centros/unidades; además de mantener una estructura 
organizacional, cuentan con un plan o programas de desarrollo profesional, y un conjunto de documentos que describen los procesos que realizan. Además, cuentan con equipos de trabajo, cuyo número de integrantes varía desde cuatro a quince personas. Esta manera de organización puede favorecer la gestión de los programas de desarrollo profesional, ya que las instituciones de educación superior cuentan con centros, cuya función principal se centra en esta actividad, lo que coincide con lo expuesto por Zabalza (2011a).

En la dimensión (2), Planificación y diseño, se analizaron varios elementos: necesidades, objetivos, contenidos, actividades, recursos y medios que se consideran para la ejecución de un programa de desarrollo profesional, haciendo referencia lo manifestado por Tejada Fernández y Navío Gámez (2007): “Justificar la necesidad de planificar la formación puede parecer una perogrullada; ahora bien, si profundizamos en esta justificación, veremos que hay que atar muchos cabos, definir muchos conceptos y poner sobre la mesa muchas concepciones" (p. 155).

En cuanto a las necesidades que dan origen a las formaciones, todas las instituciones de educación superior consideran las necesidades de formación, propias de cada universidad, y las enfocadas al cumplimiento del Artículo 88 del Reglamento de Régimen Académico, de noviembre del 2013, aprobado por el CES. Mientras que, los estudios de necesidades de formación, resultados de evaluación del docente y el perfil del docente son considerados con diferentes frecuencias (ver figura 5).

\section{Figura 5}

Necesidades consideradas para el diseño de un Programa de Desarrollo Profesional

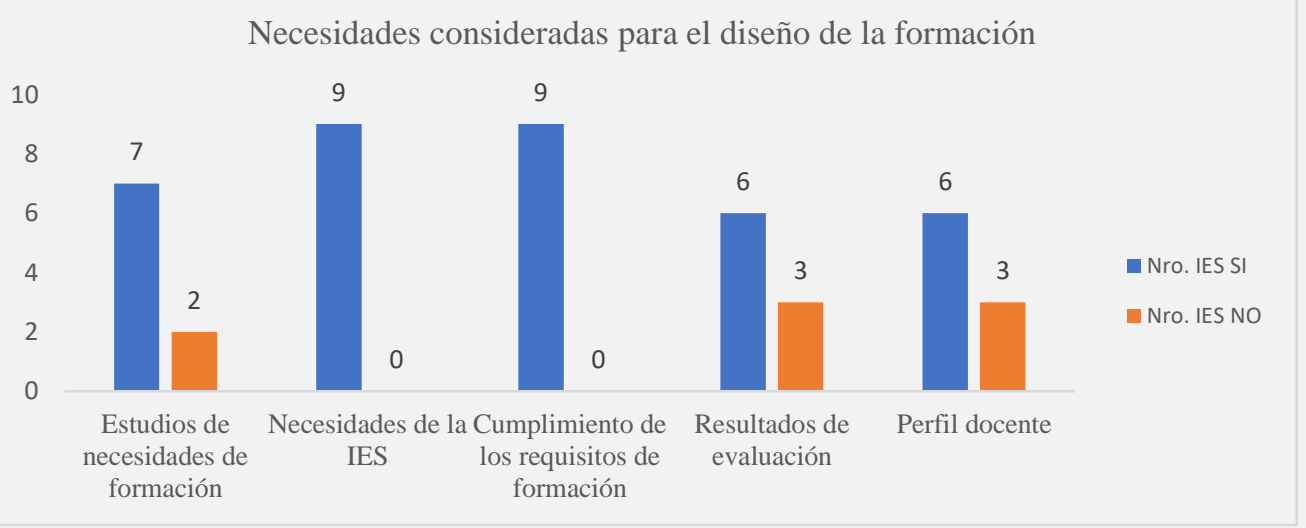

Nota: Elaboración propia 
Por medio de la revisión de los planes estratégicos y planes operativos anuales de las nueve instituciones de educación superior, se identificó que en todas existen declaraciones sobre el interés por la formación del profesorado. La manifestación más frecuente está relacionada con el fortalecimiento de las capacidades del profesorado en el ámbito pedagógico. En siete instituciones de educación superior existen declaraciones de apoyo a la formación de cuarto nivel del profesorado en maestrías y/o doctorados, lo que ayudará al profesorado a cumplir el requisito necesario para ser profesor universitario; o, en el caso de titularse como doctor, a su promoción como docente Principal.

El 33,33 \% de las instituciones de educación superior consideran algunas características del perfil de los docentes para el diseño de las formaciones: cargos de gestión, años de experiencia, categoría; mientras que el 66,67\% diseñan sin considerar diferentes perfiles.

Tanto en las necesidades de formación como en las declaraciones de los objetivos, se identifica con frecuencia baja el enfoque del programa de formación para mejorar el aprendizaje de los estudiantes, puede ser porque este elemento está asumido dentro del fortalecimiento del ejercicio de la docencia; sin embargo, centrar los esfuerzos para mejorar el aprendizaje de los estudiantes es una necesidad presente en todas las instituciones de educación superior y un objetivo a considerar para el diseño de las acciones de formación.

Sobre las temáticas abordadas, las más frecuentes están orientadas al desarrollo de metodologías de enseñanza-aprendizaje, modelo académico y herramientas de investigación. El porcentaje de distribución de estas temáticas es variado en la mayoría de las instituciones de educación superior. Los programas de desarrollo profesional establecen, en la fase de diseño, las temáticas a ofertar, según el perfil del docente, y conceder la misma importancia a las tres dimensiones críticas de la docencia: planificación, desarrollo y evaluación. Además, se observa que los programas de desarrollo profesional no desarrollan temáticas relacionadas con la motivación del profesorado, siendo esto una debilidad, ya que los autores mencionados (Kennedy, 2016; Knight, 2005; Lipowsky, 2011; Richter et al., 2019) han evidenciado en sus estudios que esta variable es determinante para el éxito del aprendizaje del profesorado y desarrollo de los programas de desarrollo profesional. 
En la mayoría de las instituciones de educación superior se distinguen dos etapas en las formaciones: inicial y continua o permanente. En el estudio se identificó pocas acciones de formación inicial; las más frecuentes se enfocan en charlas informativas y/o a inducción del modelo académico, con pocas horas de duración. Este tipo de formación mantiene estructuras básicas y su organización es aislada de las acciones de formación permanente. Considerando la propuesta de Vaillant (2016), las instituciones de educación superior deben potenciar la formación inicial en su institución, ya que es una etapa clave para garantizar el ejercicio profesional.

Se observó debilidad en el diseño de las acciones de formación permanente, siendo evidente que las instituciones de educación superior deben mejorar este aspecto, considerando que, de acuerdo con Imbernón (2020) este tipo de formación es una necesidad creciente, por lo que es reconocida como un derecho y un deber que se extiende a todas las funciones que pueden ejercer los docentes.

En la dimensión (3) Desarrollo y puesta en marcha, se analizó los procesos de difusión y orientaciones dadas al profesorado sobre las características de la formación, duración, tipo de participación del profesorado y metodología de evaluación.

La mayoría de las instituciones de educación superior se centran en la oferta de capacitaciones, cursos, talleres aislados, sin existir una coordinación entre los objetivos y los resultados que se persiguen. Sería importante que el diseño de los programas de desarrollo profesional guarde relación con la lógica de contextualización del currículo y que estén interrelacionados para obtener resultados comunes e integrados para gestionar de manera eficiente el proceso de enseñanza (Imbernón, 2011; Tejada Fernández y Navío Gámez, 2007; Yániz Álvarez, 2008).

Las formaciones consideran diferentes ratios de participación, siendo el más frecuente de 30 a 40 profesores por curso teórico, y de 25 profesores por curso práctico. En cuanto a la duración, las formaciones se organizan frecuentemente entre 20 y 40 horas de duración. El mayor porcentaje de las instituciones de educación superior organiza formaciones de 40 horas. Sobre la difusión de las acciones de formación que se ofertan, el $100 \%$ de las IES utilizan el correo electrónico como principal medio para comunicar al profesorado la realización de las acciones de formación.

Sobre los mecanismos establecidos para evaluar la participación de los docentes en cada una de las formaciones, el 44,44\% de las instituciones de educación superior tiene establecido, 
como obligatoriedad, que las formaciones incorporen mecanismos de evaluación a los docentes. Algunas universidades, en porcentaje menor, solicitan informes al formador, en relación con el desarrollo de la formación, los resultados logrados y propuestas de mejora. Otra alternativa frecuente son las encuestas aplicadas al profesorado para recabar información sobre las percepciones de la calidad de la formación y los resultados de aprendizaje alcanzados.

La participación del profesorado en las acciones de formación es voluntaria, salvo algunas capacitaciones que se han establecido como obligatorias. Sobre este aspecto los responsables del diseño de los programas de desarrollo profesional podrían establecer propuestas equilibradas que garanticen y motiven la participación voluntaria de todo el profesorado; pues es frecuente que los profesores que necesitan la formación son los menos implicados en este tema. Zabalza (2013) propone aplicar en el programa de desarrollo profesional las tres condiciones del aprendizaje: (a) evitar los obstáculos frecuentes que se escuchan del profesorado: no existe el tiempo suficiente, la institución no asigna los recursos necesarios, entre otros; (b) estimular y motivar al profesorado para que encuentre sentido y utilidad a la formación; y (c) presionar de manera directa o indirecta esta participación, por medio de alguna estrategia que utilice la institución. Por otra parte, se podría considerar el ejemplo de países como Holanda, Dinamarca, Suiza, Irlanda, entre otros, donde se han introducido, a nivel de normativa pública, la exigencia de que el profesorado universitario cumpla con horas de formación (Ruè, 2015).

Finalmente, la dimensión (4) Seguimiento y gestión de la calidad, integra diferentes criterios que hacen referencian a los mecanismos de seguimiento y evaluación de los programas de desarrollo profesional. Además, se analizó la creación de espacios comunes para el intercambio de experiencias y recursos útiles para el proceso de enseñanza.

En ninguna institución de educación superior existen estrategias formalmente establecidas para evaluar la calidad, los resultados y la aplicabilidad en el aula de la transferencia de la formación, siendo esta una tarea de gran importancia para medir los logros alcanzados del programa de desarrollo profesional y hacer un análisis del costo-beneficio. Varios autores (Cano, 2016; Feixas et al., 2013; Monereo, 2014) manifiestan que el impacto de los resultados de la formación en la evaluación del profesorado es un tema poco abordado hasta la actualidad, ya que la mayoría de las investigaciones se han centrado en diagnosticar las percepciones del profesorado 
sobre el desarrollo y calidad de las formaciones recibidas; sin embargo, existen algunos modelos o propuestas para realizar estas evaluaciones.

Realizando un análisis general de las cuatro dimensiones, se observa que la cultura de formación y aprendizaje permanente debe ser potenciada en cada instituciones de educación superior; según las manifestaciones de los entrevistados, el profesorado está comprometido con su formación; las universidades deberán implementar estrategias para mantener, valorar y estimular el compromiso del profesorado con el desarrollo institucional y su propio crecimiento profesional, lo que concuerda con las propuestas de varios autores, revisados en el marco teórico (Kennedy, 2016; Knight, 2005; Lipowsky, 2011; Richter et al., 2019).

Una de las implicaciones importantes para analizar los resultados de las acciones de formación se puede enfocar en el análisis del entorno de aprendizaje; es decir, todo aquello que está alrededor del proceso de aprendizaje de las personas, en general, y de los profesores, en particular, como actividades, herramientas, espacios, ambientes, entre otros (Fernández March, 2020). Es por esto que la presente investigación ofrece una ventaja al contar con elementos contextualizados y propios del entorno de cada instituciones de educación superior; aspectos claves para plantear las mejoras pertinentes, de acuerdo a la realidad, lo que está en consonancia con lo mencionado por Fernández y Paricio (2019), quienes indican que esto contribuye a generar condiciones propicias para afrontar eficazmente la formación de los docentes y el desarrollo educativo.

Por otra parte, los resultados de la presente investigación apoyan el trabajo desarrollado por los responsables de la formación del profesorado de las distintas universidades, ya que permiten realizar análisis integrales de este ámbito, comparando las buenas prácticas que se están desarrollando en las instituciones de educación superior, en tanto, hasta la fecha, se encuentran pocos estudios y de casos puntuales.

\section{Conclusiones}

Es importante que las instituciones de educación superior establezcan normas y políticas para fomentar y apoyar la formación pedagógica del profesorado, de cara a la institucionalización de los programas de desarrollo profesional; además, los mismos deben integrar las diferentes 
propuestas de formación, evitando la oferta de cursos/talleres/capacitaciones en forma aislada y fragmentada.

Además del fomento al desarrollo profesional del docente, de manera institucional, algunos estudios demuestran que la formación tiene éxito cuando cuenta con la motivación y la participación voluntaria del profesorado; por tanto, su éxito puede depender de la necesidad, interés y compromiso que manifieste el profesor por la calidad de la institución y su propio desarrollo profesional.

La formación inicial debe ser considerada como el punto de partida de la actividad docente y debe sembrar los cimientos necesarios para desarrollar el perfil del docente requerido por las instituciones de educación superior, así como para aportar en la construcción de la identidad del profesorado, teniendo presente que la oferta de formación inicial debe relacionarse con la permanente.

Por otra parte, debe existir una correcta diversificación de contenidos y estrategias en el diseño de las formaciones; y con base en los objetivos planteados se deberá decidir las temáticas que la institución necesita que el profesorado desarrolle.

Es fundamental que las instituciones de educación superior determinen mecanismos para medir la transferencia del aprendizaje desarrollado, lo cual ayudará a la evaluación de sus resultados y a proponer acciones correctivas; además, transferir los resultados a la práctica docente y medir el grado de aplicabilidad de la formación será un buen estímulo para el profesor. Es por esto por lo que se sugiere valorar los resultados de los programas de desarrollo profesional y su transferencia en el aprendizaje de los estudiantes, como del propio profesorado, y los beneficios obtenidos en la institución.

Finalmente, se debe considerar que las acciones establecidas en algunas universidades pueden servir de referencia para otras interesadas en la formación de su profesorado, observando algunos indicadores que se podrían mejorar; por tanto, se invita a continuar la investigación científica en este ámbito. 


\section{Referencias}

Asamblea de la República del Ecuador. (12 de octubre de 2010). Ley Orgánica de Educación Superior 0/2010. Registro Oficial 298 1-39.

Boéssio, B., y Portella, M. (2009). Docencia universitaria: formación y aprendizaje en el posgrado en educación. Revista de La Educación Superior, 38(151), 163-170. http://www.redalyc.org/articulo.oa?id=60416811009

Cabrera, A. (2002). Recursos humanos y formación en las organizaciones. En P. Pineda (Ed.), Pedagogía Laboral (pp. 131- 146). Ariel.

Cano, E. (2016). Factores favorecedores y obstaculizadores de la transferencia de la formación del profesorado en educación superior. REICE. Revista Iberoamericana sobre Calidad, $\begin{array}{lllll}\text { Eficacia } & y & \text { Cambio } & \text { en } & \text { 133-150. }\end{array}$ https://doi.org/http//doi.org/10.15366/reice2016.14.2.008

Cardeño Espinosa, J., Muñoz Marín, L. G., Ortiz Alzate, H. D., y Alzate Osorno, N. C. (2017). La incidencia de los Objetos de Aprendizaje interactivos en el aprendizaje de las matemáticas básicas, en Colombia. Trilogía Ciencia Tecnología Sociedad, 9(16), 63-84. https://doi.org/10.22430/21457778.182

Consejo de Educación Superior -CES-. (31 de octubre de 2012). Reglamento de Carrera y Escalafón del Profesor e Investigador del Sistema de Educación Superior. Registro Oficial SO-037-No.265-2012.

Consejo de Educación Superior -CES-. (2013). Reglamento de Régimen Académico. Registro Oficial SE· $13 \cdot$ No.051·2013.

Consejo de Evaluación, Acreditación y Aseguramiento de la Calidad de la Educación Superior CEAACES-. (20 de noviembre de 2013). Reglamento para la determinación del proceso de evaluación, acreditación y categorización de las universidades y escuelas politécnicas y de su situación académica e institucional. Registro Oficial 001-0074-CEAACES-2013.

Day, C. (2005). Formar Docentes. Cómo, cuándo y en qué condiciones aprende el profesorado. NARCEA.

Day, C., \& Sachs, J. (2004). Professionalism, performativity and empowerment: discourses in the politics, policies and purposes of continuing professional development. En C. Day y J. Sachs 
(Eds.), International Handbook on the Continuing Professional Development of Teachers (pp. 3-32). Open University Press, McGraw-Hill Education.

De Ketele, J.-M. (2003). La formación didáctica y pedagógica de los profesores universitarios: luces y sombras. Revista de Educación, (331), 143-169. http://www.educacionyfp.gob.es/dam/jcr:6fc1750b-4e67-4f88-9ccabad8d18af51f/re3310711294-pdf.pdf

De la Torre, S. (2000). Estrategias Didácticas Innovadoras. Octaedro.

Fabara Garzón, E. (2016). La formación y el ejercicio de la docencia universitaria en Ecuador. Desafíos. Alteridad. Revista de Educación, 11(2), 171-181. https://doi.org/http//doi.org/10.17163/alt.v11n2.2016.03

Feixas, M., Duran, M., Fernández, I., Férnandez March, A., García, M. J., Márquez, M. D., Pineda, P., Quesada, C., Sabaté, S., Tomás, M., Zellweger, F., y Lagos, P. (2013). ¿Cómo medir la transferencia de la formación en Educación Superior?: el Cuestionario de Factores de Transferencia. Revista de Docencia Universitaria, 11(3), 219-248. https://doi.org/10.4995/redu.2013.5527

Fernández March A. (2006). La estructura y contenido de la formación del profesorado en las universidades. En A. Alías, C. Gil, A. Riscos, M. Valcarcel, y E. Vicario, La Formación del profesorado universitario (pp. 35-50). Editorial Universidad de Almería.

Fernández March, A. (2008). La formación inicial del profesorado universitario: el título de Especialista Universitario en Pedagogía Universitaria de la Universidad Politécnica de Valencia. Revista Interuniversitaria de Formación del Profesorado, 63(22), 161-187. https://core.ac.uk/download/pdf/41576304.pdf

Fernández March, A. (2009). La gestión de la formación del profesorado en la Universidad. Teoría de La Educación. Revista Interuniversitaria, 20, 275-312. http://revistas.usal.es/index.php/1130-3743/article/view/993

Fernández March, A. (2020). Entornos de aprendizaje para el desarrollo profesional docente. REDU: Revista de Docencia Universitaria, 18(1), 169-191. https://doi.org/10.4995/redu.2020.13145

Fernández, A., y Paricio, J. (2019). Entornos de aprendizaje como espacios para la acción, interacción, autorregulación y elaboración personal del conocimiento. En J. Paricio, A. 
Fernández y I. Fernández (Ed.), Cartografía de la buena docencia. Un Marco para el desarrollo del profesorado basado en la investigación. Narcea.

Gómez, D. (2009). La creación y gestión del conocimiento en organizaciones educativas:

Barreras y Facilitadores (Tesis de doctorado). Universidad Autónoma de Barcelona. https://ddd.uab.cat/pub/tesis/2009/hdl_10803_327017/drg1de1.pdf

Herdoíza, M. (2015). Construyendo Igualdad en la Educación Superior. SENESCYT - UNESCO. https://www.educacionsuperior.gob.ec/wpcontent/uploads/downloads/2018/11/Construyendo-igualdad-en-la-educacionsuperior_nov_2018.pdf

Imbernón, F. (2011). La formación pedagógica del docente universitario. Pedagogía y Saberes, 21, 29-35. https://www.redalyc.org/articulo.oa?id=1171/117121313005

Imbernón, F. (2020). Desarrollo personal, profesional e institucional y formación del profesorado. Algunas tendencias para el siglo XXI. Revista Currículum, 33, 49-67. https://riull.ull.es/xmlui/bitstream/handle/915/19619/Q_33_\%282020\%29_04.pdf?sequen $\underline{\mathrm{ce}=1 \& \text { is Allowed }=\mathrm{y}}$

Imbernón, F., Silva, P., y Guzmán, C. (2011). Competencias en los procesos de enseñanzaaprendizaje virtual y semipresencial. Comunicar, 18(36), 107-114. https://doi.org/10.3916/C36-2011-03-01

Institute of Personal and Development. (2000). Designing Learning Programmes. EPISE, S.A. Kane, R.G., Sandretto, S., \& Heath, C.J. (2002). Telling half the story: A critical review on the teaching beliefs and practices of university academics. Review of Educational Research. 72(2), 177-228. https://doi.org/10.3102/00346543072002177

Kennedy, M. (2016). How Does Professional Development Improve Teaching? Review of Educational Research, 86(4). https://doi.org/10.3102/0034654315626800

Knight, P. T. (2005). El profesorado de Educación Superior. NARCEA S.A.

Korthagen, F. A. J. (2004). In search of the essence of a good teacher: Towards a more holistic approach in teacher education. Teaching and Teacher Education, 20(1), 77-97. https://talispiritualeducation.org.il/korthagen-f-a-j-2004-in-search-of-the-essence-of-agood-teacher-towards-a-more-holistic-approach-in-teacher-education-teaching-andteacher-education-20-77-97-2/ 
Korthagen, F. A. J. (2010). La práctica, la teoría y la persona en la formación del profesorado. Revista Interuniversitaria de Formación del Profesorado, 68(68), 83-102. https://dialnet.unirioja.es/servlet/articulo?codigo $=3276048$

Leandro da Silva, L., y Tejada Fernández, J. (2016). La formación del profesorado universitario en Cataluña y São Paulo: dilemas y desafíos. Revista Española de Educación Comparada, 27, 193-213. https://doi.org/10.5944/reec.27.2016.15989

Lipowsky, F. (2011). Theoretische Perspektiven und empirische Befunde zur Wirksamkeit von Lehrerfort-und-weiterbildung. En E. Terhart, H. Bennewitz \& M. Rothland (Red.), Handbuch der Forschung zum Lehrerberuf (pp. 398-417). Waxmann.

Lopera, M., Arias, V., Jiménez, M., Ospina, D., y Valderrama, Á. (2021). Aportes de la revisión de literatura al diseño de una ruta de apropiación TIC, vinculada con el modelo tecnológicopedagógico-disciplinar. Revista Virtual Universidad Católica del Norte, (62), 276-307. https://www.doi.org/10.35575/rvucn.n62a11

López, F. (2005). Metodología participativa en la Enseñanza Universitaria. NARCEA S.A.

Marcelo, C. (2007). Programas de Formación Docente a través de Estrategias de Aprendizaje Abierto y a Distancia. OREAL - UNESCO.

Marcelo, C., y Vaillant, D. (2009). Desarrollo profesional docente. ¿Cómo se aprende a enseñar? NARCEA S.A.

Marcelo, C., y Zapata, M. (2008). Cuestionario para la evaluación. "Evaluación de la calidad para programas completos de formación docente a través de estrategias de aprendizaje abierto y a distancia”. Metodología de uso y descripción de indicadores. RED, Revista de Educación $\quad$ a Distancia, 31. https://www.researchgate.net/publication/28243320_Cuestionario_para_la_evaluacion_E $\underline{\text { valuacion_de_la_calidad_para_programas_completos_de_formacion_docente_a_traves_d }}$ e_estrategias_de_aprendizaje_abierto_y_a_distancia_Metodologia_de_uso_y_descripcion de

Martínez Villalobos, G., Arciniegas, A. M., y Lugo González, C. A. (2016). Formación docente en TIC con el Centro de Innovación Educativa CIER-SUR. Trilogía Ciencia Tecnología Sociedad, 8(14), 65-80. https://doi.org/10.22430/21457778.417

Mateo, J. (2012). La formación de formadores en la Educación Superior. Training of trainers in 
Higher Education Introducción Investigación y docencia, 10(2), 211-223. https://www.researchgate.net/publication/321737296_La_Formacion_de_Formadores_en _la_Educacion_Superior

Mayor Ruiz, C. (2007). El asesoramiento pedagógico para la formación docente del profesorado universitario. Secretariado de publicaciones de la Universidad de Sevilla.

Mayor, C., Marcelo, C., Sánchez, M., Murillo, P., y Hernández, E. (2003). Enseñanza y aprendizaje en la Educación Superior. OCTAEDRO.

Melo, G., \& Campos, V. (2019). University Pedagogy: for an institutional teaching development policy in higher education. Cadernos de Pesquisa, 49(173), 44-62. https://doi.org/10.1590/198053145897

Monereo, C. (2014). La investigación en la formación del profesorado universitario: hacia una perspectiva integradora. Infancia y Aprendizaje, 36(3), 281-291. http://doi.org/10.1174/021037013807533052

Reis-Jorge, J., Ferreira, M., y Olcina-Sempere, G. (2020). La figura del profesorado-investigador en la reconstrucción de la profesionalidad docente en un mundo en transformación. Revista Educación, 44(1), 509-519. https://dialnet.unirioja.es/servlet/articulo?codigo=7214946

Richter, D., Kleinknecht, M., \& Gr, A. (2019). What motivates teachers to participate in professional development? An empirical investigation of motivational orientations and the uptake of formal learning opportunities. Teaching and Teacher Education, 86, 1-10. https://doi.org/10.1016/j.tate.2019.102929

Restrepo, R., y Stefos, E. (2017). Atlas del derecho a la educación en los años de la Revolución Ciudadana. Universidad Nacional de Educación.

Roldán, N. (2018). Dos décadas potenciando el tipo de docente en y para la virtualidad. Revista $\begin{array}{llll}\text { Reflexiones } & y & \text { Saberes, } & \text { 73-80. }\end{array}$ https://revistavirtual.ucn.edu.co/index.php/RevistaRyS/article/view/972/1421

Ruè, J. (2015). El desarrollo profesional docente en Educación Superior: agenda, referentes y propuestas para su adopción. Revista de Docencia Universitaria, 13, 217-236. https://doi.org/10.4995/redu.2015.5461

Saavedra Bautista, C. E. (2018). La formación de maestros en el marco de apuestas tecnológicas emergentes. Revista Virtual Universidad Católica del Norte, (53), 2-17. 


\section{https://revistavirtual.ucn.edu.co/index.php/RevistaUCN/article/view/961/1409}

Secretaría Nacional de Educación Superior Ciencia, Tecnología e Innovación -SENESCYT(2018). Información general. https://www.educacionsuperior.gob.ec/

Tapia Henríquez, M. (2013). Sentido y significado de la experiencia formativa del profesorado principiante de Pedagogía en español de la Universidad de Concepción (tesis doctoral). Universidad de Barcelona.

Tejada Fernández, J. (2000). Estrategias Didácticas Innovadoras. Recursos para la Formación y el Cambio. Octaedro.

Tejada Fernández, J. (2013). Professionalisation of Teaching in Universities: Implications from a Training Perspective. RUSC. Universities and Knowledge Society Journal, 10(1), 170-184358. http://doi.org/10.7238/rusc.v10i1.1471

Tejada Fernández, J., y Navío Gámez, A. (2007). Elaboración de planes, programas y cursos de formación. En J. Tejada, y V. Giménez, Formación de Formadores (pp. 153-205). Thomson Editores.

Vaillant, D. (2016). El fortalecimiento del desarrollo profesional docente: una mirada desde latinoamérica. Journal of Supranational Policies of Education, (5), 5-21. https://doi.org/10.15366/jospoe2016.5

Villa, A. (2001). Evaluación de la función docente y desarrollo del profesorado. En C. Marcelo (Ed.), La función docente (pp. 171-201). Síntesis, S.A.

Yániz Álvarez, C. (2008). Las competencias en el currículo universitario. Revista de Docencia Universitaria, 4, 1-14. http://www.um.es/ead/Red_U/m1/yaniz.pdf

Yunga, D. C., Loaiza, M. I., Ramón, L. N., y Puertas, L. (2016). Enfoques de la Enseñanza en Educación Universitaria: Una exploración desde la perspectiva Latinoamericana, Profesorado. Revista de Currículum y Formación del Profesorado, 313-333. https://recyt.fecyt.es/index.php/profesorado/article/view/54600

Zabalza M. (2011a). Evaluación de los planes de formación docente de las universidades. Educar, 47(1), 181-197. http://dialnet.unirioja.es/servlet/articulo?codigo=3696862\&info=resumen\&idioma=ENG

Zabalza, M. (2011b). Formación del profesorado universitario: mejorar a los docentes para mejorar la docencia. Educação (UFSM), 36(3), 397-423. https://doi.org/10.5902/198464442969 


\section{Análisis de programas}

Análisis de programas | Revista Virtual Universidad Católica del Norte, 63, 161-195

ISSN: 0124-5821 (En línea)

Zabalza, M. (2013). La formación del profesorado universitario. Revista de Docencia Universitaria, 11(3),

$11-15$.

https://polipapers.upv.es/index.php/REDU/article/view/5515/5506 